\title{
SOME TIME STEPPING METHODS FOR FRACTIONAL DIFFUSION PROBLEMS WITH NONSMOOTH DATA
}

\author{
YAN YANG, YUBIN YAN AND NEVILLE J. FORD *
}

\begin{abstract}
We consider error estimates for some time stepping methods for solving fractional diffusion problems with nonsmooth data in both homogeneous and inhomogeneous cases. McLean and Mustapha [19] (Time-stepping error bounds for fractional diffusion problems with non-smooth initial data, Journal of Computational Physics, 293(2015), 201-217) established an $O(k)$ convergence rate for the piecewise constant discontinuous Galerkin method with nonsmooth initial data for the homogeneous problem when the linear operator $A$ is assumed to be self-adjoint, positive semidefinite and densely defined in a suitable Hilbert space, where $k$ denotes the time step size. In this paper, we approximate the Riemann-Liouville fractional derivative by Diethelm's method (or $L 1$ scheme) and obtain the same time discretisation scheme as in McLean and Mustapha [19]. We first prove that this scheme has also convergence rate $O(k)$ with nonsmooth initial data for the homogeneous problem when $A$ is a closed, densely defined linear operator satisfying some certain resolvent estimates. We then introduce a new time discretization scheme for the homogeneous problem based on the convolution quadrature and prove that the convergence rate of this new scheme is $O\left(k^{1+\alpha}\right), 0<\alpha<1$ with the nonsmooth initial data. Using this new time discretization scheme for the homogeneous problem, we define a time stepping method for the inhomogeneous problem and prove that the convergence rate of this method is $O\left(k^{1+\alpha}\right), 0<\alpha<1$ with the nonsmooth data. Numerical examples are given to show that the numerical results are consistent with the theoretical results.
\end{abstract}

Key words. fractional diffusion problem, nonsmooth data, error estimates, Laplace transform

AMS subject classifications. 26A33, 65M06, 65M12, 65M15, 35R11

1. Introduction. Consider the following time fractional diffusion problem, with $0<\alpha<1,[18,(4)],[9]$,

$$
{ }_{0}^{C} D_{t}^{\alpha} u(t)+A u(t)=f(t), \quad \text { for } 0<t \leq T, \quad \text { with } u(0)=u_{0},
$$

where $f$ is a given function, $u_{0}$ is the initial value and ${ }_{0}^{C} D_{t}^{\alpha} u(t)$ denotes the Caputo fractional derivative defined by

$$
{ }_{0}^{C} D_{t}^{\alpha} u(t)=\frac{1}{\Gamma(1-\alpha)} \int_{0}^{t}(t-s)^{-\alpha}\left(\frac{d u(s)}{d s}\right) d s .
$$

Here $A$ is a closed, densely defined linear operator and the resolvent satisfies, for some $\pi / 2<\theta_{0}<\pi$, see Lubich et al. [17], Thomée [25],

$$
\left\|(z I+A)^{-1}\right\| \leq C|z|^{-1} \quad \text { for } z \in \Sigma_{\theta_{0}}=\left\{z \neq 0:|\arg \mathrm{z}|<\theta_{0}\right\} .
$$

For example, $A$ may be the Laplacian $-\Delta$ on a polyhedral domain $\Omega \subset \mathbb{R}^{d}(d=$ $1,2,3)$ with the homogeneous Dirichlet boundary condition. In this case (1.3) holds for all $\theta_{0} \in(\pi / 2, \pi)$, see Jin et al. [12, (1.3)].

In our analysis, we will choose $\theta>\pi / 2$ close to $\pi / 2$ such that $\theta_{0}>\theta$ which implies that $z^{\alpha} \in \Sigma_{\theta_{0}}$ for any $z \in \Gamma=\Gamma_{\theta}=\{z:|\arg z|=\theta\}$, since $\arg \left(z^{\alpha}\right)=\alpha \theta<\theta<\theta_{0}$

* Yan Yang: Department of Mathematics, Lvliang University, Lishi, 033000, P.R. China, (enn_yang@163.com), Yubin Yan: Department of Mathematics, University of Chester, Thornton Science Park, Pool Lane, Ince, CH2 4NU, UK, (y.yan@chester.ac.uk), Neville J. Ford: Department of Mathematics, University of Chester, Thornton Science Park, Pool Lane, Ince, CH2 4NU, UK, (njford@chester.ac.uk). Dr. Yubin Yan is the corresponding author. 
for $0<\alpha<1$. Hence there exists a constant $C$ which depends only on $\theta$ and $\alpha$ such that, see Jin et al. [10, (2.3)],

$$
\left\|\left(z^{\alpha} I+A\right)^{-1}\right\| \leq C|z|^{-\alpha}, \quad \forall z \in \Gamma_{\theta}=\{z:|\arg z|=\theta\} .
$$

We also need to restrict $\theta$ further and choose $\theta>\pi / 2$ close to $\pi / 2$ such that $z_{k}^{\alpha} \in \Sigma_{\theta_{0}}$ for $z \in \Gamma_{\theta}$ which implies that $\left(z_{k}^{\alpha} I+A\right)^{-1}$ exists, where $z_{k}=\frac{\delta\left(e^{-z k}\right)}{k}$ is defined in (2.11) or (2.36) below.

Let us first consider the homogeneous problem (1.1), that is, $f=0$. It is well known that the homogeneous problem (1.1) is equivalent to, [19]

$$
u_{t}+{ }_{0}^{R} D_{t}^{1-\alpha} A u=0, \quad \text { for } 0<t \leq T, \quad \text { with } u(0)=u_{0},
$$

where $u_{t}$ denotes the time derivative and ${ }_{0}^{R} D_{t}^{\alpha} u(t)$ denotes the Riemann-Liouville fractional derivative defined by

$$
{ }_{0}^{R} D_{t}^{1-\alpha} u(t)=\frac{1}{\Gamma(\alpha)} \frac{d}{d t} \int_{0}^{t}(t-s)^{\alpha-1} u(s) d s .
$$

The time discretisation of (1.5) has been considered by many authors. Under the assumptions that the solution of (1.5) is sufficiently smooth, e.g., $u \in C^{2}[0, T]$ in the time variable, the optimal order error estimates uniformly in $t$ for the time discretisation schemes of (1.5) can be obtained, see, for example, [1], [16], [3], [13], [20], [2], [26], [7], [14], [4], [15]. However the $C^{2}$-regularity assumption for the solution of (1.5) does not hold when the initial value $u_{0} \in L_{2}(\Omega)$. For example, Sakamoto and Yamamoto [23, Theorem 2.1] showed that the solution $u$ of (1.5) satisfies

$$
\left\|{ }_{0}^{C} D_{t}^{\alpha} u\right\|_{L_{2}(\Omega)} \leq c t^{-\alpha}\left\|u_{0}\right\|_{L_{2}(\Omega)},
$$

which implies that the Caputo derivative may not be bounded when $u_{0} \in L_{2}(\Omega)$. Hence in general $u \notin C^{2}[0, T][10]$. Therefore the optimal convergence rates of the time discretization schemes cannot be achieved uniformly in $t$ when $u_{0} \in L_{2}(\Omega)$ with uniform meshes. By using the variable time steps, uniform error estimates in $t$ can be achieved when the solution $u$ is not sufficiently smooth, see, for example, [19], [21], [22], [24]. However no error estimates with nonsmooth initial data were given in [19], [21], [22], [24]. In this paper, we will consider the time discretization schemes for (1.1) with nonsmooth initial data, at the cost of requiring a constant time step. More precisely, we will first consider the nonsmooth data error estimates for the piecewise constant discontinuous Galerkin method introduced in McLean and Mustapha [19] for solving the homogeneous problem (1.5). Then we introduce and analyze a new time discretization scheme for solving (1.5) based on the approximation of the time derivative with the backward difference formula of order 2 and the approximation of the Riemann-Liouville fractional derivative with a suitable convolution quadrature.

The discontinuous Galerkin method and the convolution quadrature method are both very popular time discretization methods for solving the time fractional partial differential equations and they have the different advantages. The advantages of the discontinuous Galerkin method are as follows: 1). the discontinuous Galerkin method is unconditionally stable even when we choose a different trial space for each time step combined with arbitrarily-spaced time levels which allows great flexibility in the choice of mesh, McLean and Mustapha [18]; 2). the error bounds of the discontinuous Galerkin method can be proved uniformly in $t$ with the variable steps 
even the derivative of the solution $u(t)$ is unbounded as $t \rightarrow 0$, McLean and Mustapha [19]. The convolution quadrature method has other advantages: 1$)$. the convolution quadrature method enables us to approximate the time derivative and the RiemannLiouville fractional derivative as a whole and the error estimates can be considered based on the resolvent bounds of the elliptic operator; 2). the error estimates depend only on the regularity of the data rather than of the solution $u(t)$, Cuesta et al. [2]; 3). it is possible to restore the convergence orders of some higher order time discretization schemes by correcting a few starting steps of the schemes when the solution $u(t)$ is not smooth, Jin et al. [12].

Let $N \geq 1$ be a positive integer and let $0=t_{0}<t_{1}<t_{2}<\cdots<t_{N}=T$ be a partition of $[0, T]$ with $k$ the time step size. Let $U^{n} \approx u\left(t_{n}\right), n=1,2, \ldots, N$ be the approximate solution of $u\left(t_{n}\right)$. McLean and Mustapha [19, (6)] define the following piecewise constant discontinuous Galerkin method for solving (1.5), with $U^{0}=u_{0}$,

$$
U^{n}-U^{n-1}+k^{\alpha} \sum_{j=1}^{n} w_{n-j} A U^{j}=0, n \geq 1,
$$

where $w_{j}, j=0,1,2, \ldots, n-1, n \geq 1$ are given by

$$
\Gamma(1+\alpha) w_{j}=\left\{\begin{array}{l}
1, \quad \text { for } j=0, \\
-2 j^{\alpha}+(j-1)^{\alpha}+(j+1)^{\alpha}, \text { for } j=1,2, \ldots, n-1 .
\end{array}\right.
$$

Assume that $A$ is self-adjoint, positive semidefinite and densely defined operator in $H=L_{2}(\Omega)$, with a complete orthonormal eigensystem. Let $U^{n}$ and $u\left(t_{n}\right), n=$ $1,2, \ldots, N$ be the solutions of (1.6) and (1.5), respectively. McLean and Mustapha $\left[19\right.$, Theorem 5] proved the following error estimates with nonsmooth data $u_{0} \in H$ :

$$
\left\|U^{n}-u\left(t_{n}\right)\right\| \leq c k t_{n}^{-1}\left\|u_{0}\right\| .
$$

Starting from the scheme (1.6), we will consider the following issues in this paper:

- We show that the piecewise constant discontinuous Galerkin method introduced in McLean and Mustaph [19] for the homogeneous problem (1.5) can also be derived by approximating the Riemann-Liouville fractional derivative with Diethelm's method [5] (or the $L 1$ scheme [16]).

- We show that the nonsmooth data error estimates of the numerical methods introduced in McLean and Mustaph [19] for the homogeneous problem (1.5) also hold for the general linear operator $A$ by using Laplace transform method developed in Lubich et al. [17], where $A$ is a closed, densely defined operator satisfying (1.4). In McLean and Mustaph [19], the linear operator $A$ is assumed to be self-adjoint, positive semidefinite and densely defined in $H=L_{2}(\Omega)$, with a complete orthonormal eigensystem.

- We introduce a modified piecewise constant discontinuous Galerkin method for the homogeneous problem (1.5) and prove that this method has the convergence rate $O\left(k^{1+\alpha}\right), 0<\alpha<1$ with nonsmooth initial data by using Laplace transform method.

- We introduce a new time discretization scheme for solving the inhomogeneous problem (1.1) and the error estimates with the convergence rate $O\left(k^{1+\alpha}\right)$ are proved.

The rest of the paper is organized as follows. In Section 2, we consider the error estimates for the homogeneous problems with nonsmooth initial data for the different 
time discretization schemes. In Section 3, we consider the error estimates for the inhomogeneous problem with nonsmooth initial data $u_{0}$ and some suitable $f$. Finally in Section 4, we give some numerical examples to illustrate the theoretical results developed in this paper.

Throughout, the notations $C$ and $c$, with or without a subscript, denote generic constants, which may differ at different occurrences, but are always independent of the step size $k$.

2. Homogeneous problem. In this section, we will introduce and analyze three types of time discretization schemes for solving (1.5).

2.1. A time stepping method with the convergence rate $O\left(k^{\alpha}\right), 0<\alpha<$ 1. In this section, we will consider a time stepping method for solving (1.5) which has only $O\left(k^{\alpha}\right), 0<\alpha<1$ convergence rate. We then modify this time stepping method in the subsequent subsections to obtain the time discretization schemes for solving (1.5) with the convergence rates $O(k)$ and $O\left(k^{1+\alpha}\right), 0<\alpha<1$, respectively.

At $t=t_{n}$, we approximate the time derivative by using the backward Euler method

$$
u_{t}\left(t_{n}\right)=\left(u\left(t_{n}\right)-u\left(t_{n-1}\right)\right) / k+O(k), \quad \text { as } k \rightarrow 0 .
$$

To approximate the Riemann-Liouville fractional derivative ${ }_{0}^{R} D_{t}^{1-\alpha} A u\left(t_{n}\right)$, we shall use the following Diethelm's finite difference method [5], with $u \in C^{2}[0, T ; \mathcal{D}(A)]$

$$
{ }_{0}^{R} D_{t}^{1-\alpha} A u\left(t_{n}\right)=k^{\alpha-1} \sum_{j=0}^{n} w_{n-j} A u\left(t_{j}\right)+O\left(k^{1+\alpha}\right), \quad \text { as } k \rightarrow 0,
$$

where $w_{j}, j=0,1,2, \ldots, n-1$ are given by (1.7) and $w_{n}$ satisfies

$$
\Gamma(1+\alpha) w_{n}=(n-1)^{\alpha}-n^{\alpha}+\alpha n^{\alpha-1} .
$$

We remark that the weights $w_{j}, j=0,1,2, \ldots, n-1, n$ in (2.1) can also be obtained by using the L1 scheme, see, for example, [16].

With $U^{n} \approx u\left(t_{n}\right)$, we define the following time discretization problem for solving (1.5), with $A u_{0} \in L_{2}(\Omega)$,

$$
U^{n}-U^{n-1}+k^{\alpha} \sum_{j=0}^{n} w_{n-j} A U^{j}=0, n \geq 1, \text { with } U^{0}=u_{0},
$$

where $w_{j}, j=0,1,2, \ldots, n-1$ are given by (1.7) and $w_{n}$ is corrected as

$$
\Gamma(1+\alpha) w_{n}=-2 n^{\alpha}+(n-1)^{\alpha}+(n+1)^{\alpha} .
$$

The reason for correcting $w_{n}$ is that we shall use the discrete Laplace transform $\tilde{w}(z)=\sum_{j=0}^{\infty} w_{j} z^{j}$ to prove the error estimates. To obtain the expression for $\tilde{w}$, we shall choose $w_{0}, w_{1}, w_{2}, \ldots, w_{n}, \ldots$ as the following

$$
\Gamma(1+\alpha) w_{0}=1, \Gamma(1+\alpha) w_{j}=-2 j^{\alpha}+(j-1)^{\alpha}+(j+1)^{\alpha}, j=1,2, \ldots, n, \ldots
$$

Theorem 2.1. Let the operator $A$ be a closed, densely defined linear operator satisfying (1.4). Let $u\left(t_{n}\right)$ and $U^{n}$ be the solutions of (1.5) and (2.3), respectively. Let $u_{0} \in L_{2}(\Omega)$. Then we have, with $0<\alpha<1$,

$$
\left\|u\left(t_{n}\right)-U^{n}\right\| \leq C\left(k^{\alpha} t_{n}^{-\alpha}+k t_{n}^{-1}\right)\left\|u_{0}\right\| .
$$


REMARK 2.2. In the time discretization scheme (2.3), we require $A u_{0} \in L_{2}(\Omega)$, i.e., the initial data $u_{0}$ is reasonably smooth. But one may use the scheme (2.3) to prove the error estimates with the nonsmooth initial data $u_{0} \in L_{2}(\Omega)$ as we will do in the proof of Theorem 2.1 below, such idea has been used in Jin et al, [12, Remark 2.4] and Lubich et al. [17, (1.8)]. The simialr remark is also for the time discretization scheme (2.29)-(2.31) below.

REMARK 2.3. We remark that the convergence rate in Theorem 2.1 is $O\left(k^{\alpha}\right), 0<$ $\alpha<1$ for $t_{n}$ not close to $t_{0}$. The similar remarks are also for other time discretization schemes discussed in Sections 2.2 and 2.3 below.

To prove Theorem 2.1, we need to show that $z_{k}^{\alpha} \in \Sigma_{\theta_{0}}$ for $z \in \Gamma=\Gamma_{\theta}=\{z$ : $|\arg z|=\theta\}$ with some $\theta>\pi / 2$ close to $\pi / 2$, where $\theta_{0} \in(\pi / 2, \pi)$ and $z_{k}$ is defined in (2.11) below. We have

LEMMA 2.4. Let $\theta>\pi / 2$ be close to $\pi / 2$. Let $z \in \Gamma_{k}$ with $\Gamma_{k}=\{z: z \in$ $\Gamma,|\Im z| \leq \pi / k\}$ where $\Gamma=\{z:|\arg z|=\theta\}$ (with $\Im z$ running from $-\infty$ to $\infty$ ). Let $z_{k}=\delta(\zeta) / k$ with $\zeta=e^{-z k}$ be defined by (2.11), where

$$
\delta(\zeta)^{\alpha}=(1-\zeta) \tilde{w}(\zeta)^{-1}
$$

and $\tilde{w}(\zeta)=\sum_{j=0}^{\infty} w_{j} \zeta^{j}$ with $w_{j}, j=0,1,2, \ldots$ defined by (2.5). Then there exists $\theta_{0} \in(\pi / 2, \pi)$ such that

$$
z_{k}^{\alpha} \in \Sigma_{\theta_{0}}, \quad \text { for all } z \in \Gamma_{\theta} \text {. }
$$

Proof. See the Appendix. $\square$

LEMMA 2.5. Let $w_{j}, j=0,1,2, \ldots, n, \ldots$ be defined as in (2.5). Then we have the following singularity expansion, with $\zeta=e^{-z k}$,

$$
(1-\zeta) \tilde{w}(\zeta)^{-1}=(z k)^{\alpha}+c_{1}(z k)^{1+\alpha}+c_{2}(z k)^{1+2 \alpha}+\ldots
$$

for some suitable constants $c_{1}, c_{2}, \ldots$.

Proof. By (5.1) and (5.2) in the Appendix, we have, with some suitable constants $c_{1}, c_{2}, \ldots$,

$$
\begin{aligned}
\tilde{w}(\zeta) & =\frac{1}{\Gamma(1+\alpha)}\left(\left(e^{-z k}\right)^{-1}-2+e^{-z k}\right) \mathrm{Li}_{-\alpha}(\zeta) \\
& =\left((z k)^{2}+\frac{1}{12}(z k)^{4}+\ldots\right)\left((z k)^{-\alpha-1}+c_{1}(z k)^{0}+c_{2}(z k)^{1}+\ldots\right) \\
& =(z k)^{1-\alpha}+c_{1}(z k)^{2}+c_{2}(z k)^{3-\alpha}+\ldots
\end{aligned}
$$

Thus

$$
\begin{aligned}
& (1-\zeta) \tilde{w}(\zeta)^{-1}=\left(1-e^{-z k}\right)\left(\tilde{w}\left(e^{-z k}\right)\right)^{-1} \\
& =\left(z k-\frac{(z k)^{2}}{2}+\frac{(z k)^{3}}{3 !}+\ldots\right)\left((z k)^{1-\alpha}+c_{1}(z k)^{2}+c_{2}(z k)^{3-\alpha}+\ldots\right)^{-1} \\
& =\left(z k-\frac{(z k)^{2}}{2}+\frac{(z k)^{3}}{3 !}+\ldots\right)(z k)^{\alpha-1}\left[1+\left(c_{1}(z k)^{1+\alpha}+c_{2}(z k)^{2}+\ldots\right)\right. \\
& \left.\quad+\left(c_{1}(z k)^{1+\alpha}+c_{2}(z k)^{2}+\ldots\right)^{2}+\ldots\right] \\
& =\left(z k-\frac{(z k)^{2}}{2}+\frac{(z k)^{3}}{3 !}+\ldots\right)(z k)^{\alpha-1}\left(1+c_{1}(z k)^{1+\alpha}+c_{2}(z k)^{2}+\ldots\right) \\
& =(z k)^{\alpha}+c_{1}(z k)^{1+\alpha}+c_{2}(z k)^{1+2 \alpha}+\ldots
\end{aligned}
$$


Together these estimates complete the proof of Lemma 2.5. $\square$

Proof. [Proof of Theorem 2.1] Let $v(t)=u(t)-u_{0}$ and $V^{n}=U^{n}-u_{0}$. It suffices to show

$$
\left\|v\left(t_{n}\right)-V^{n}\right\| \leq C\left(k^{\alpha} t_{n}^{-\alpha}+k t_{n}^{-1}\right)\left\|u_{0}\right\|
$$

which we will prove now.

Note that, by (1.5)

$$
v_{t}+{ }_{0}^{R} D_{t}^{1-\alpha} A v(t)=-{ }_{0}^{R} D_{t}^{1-\alpha} A u_{0}, \quad 0<t \leq T .
$$

Taking the Laplace transform in (2.9), we have,

$$
\hat{v}(z)=-z^{-1}\left(z^{\alpha}+A\right)^{-1} A u_{0}
$$

which implies that

$$
v(t)=-\frac{1}{2 \pi i} \int_{\Gamma} e^{z t} z^{-1}\left(z^{\alpha}+A\right)^{-1} A u_{0} d z
$$

where $\Gamma=\Gamma_{\theta}=\{z:|\arg z|=\theta\}$, for some $\theta>\frac{\pi}{2}$ determined by Lemma 2.4.

Further we note that $V^{n}, n=1,2,3, \ldots$ satisfy, by $(2.3)$, with $V^{0}=0$,

$$
V^{n}-V^{n-1}+k^{\alpha} \sum_{j=0}^{n} w_{n-j} A V^{j}=-k^{\alpha} \sum_{j=0}^{n} w_{n-j} A u_{0}, n \geq 1
$$

Thus we have

$$
\sum_{n=1}^{\infty}\left(V^{n}-V^{n-1}\right) \zeta^{n}+\sum_{n=1}^{\infty} k^{\alpha}\left(\sum_{j=0}^{n} w_{n-j} A V^{j}\right) \zeta^{n}=-\sum_{n=1}^{\infty} k^{\alpha}\left(\sum_{j=1}^{n} w_{n-j} A u_{0}+w_{n} A u_{0}\right) \zeta^{n} .
$$

With $\tilde{V}(\zeta)=\sum_{n=0}^{\infty} V^{n} \zeta^{n}$, we have

$$
\begin{aligned}
(1-\zeta) \tilde{V}(\zeta)+k^{\alpha} \tilde{w}(\zeta) A \tilde{V}(\zeta) & =-k^{\alpha}\left(\tilde{w}(\zeta) \frac{\zeta}{1-\zeta}+\tilde{w}(\zeta)-w_{0}\right) A u_{0} \\
& =-k^{\alpha}\left(\tilde{w}(\zeta) \frac{1}{1-\zeta}-w_{0}\right) A u_{0}
\end{aligned}
$$

With $\delta(\zeta)^{\alpha}=(1-\zeta) \tilde{w}(\zeta)^{-1}$ defined by $(2.7)$, see [17], we have

$$
\left(\frac{\delta(\zeta)}{k}\right)^{\alpha} \tilde{V}(\zeta)+A \tilde{V}(\zeta)=-\left(\frac{\delta(\zeta)}{k}\right)^{\alpha}\left(k^{\alpha}(1-\zeta)^{-1}\right)\left(\tilde{w}(\zeta) \frac{1}{1-\zeta}-w_{0}\right) A u_{0}
$$

Therefore we get

$$
\begin{aligned}
\tilde{V}(\zeta) & =-\left(\left(\frac{\delta(\zeta)}{k}\right)^{\alpha}+A\right)^{-1}\left(\tilde{w}(\zeta)^{-1}\left(\tilde{w}(\zeta) \frac{1}{1-\zeta}-w_{0}\right) A u_{0}\right) \\
& =-\left(\left(\frac{\delta(\zeta)}{k}\right)^{\alpha}+A\right)^{-1}\left(\frac{1}{1-\zeta}-w_{0} \tilde{w}(\zeta)^{-1}\right) A u_{0}
\end{aligned}
$$

Further we denote

$$
z_{k}=\frac{\delta(\zeta)}{k}
$$


By Lemma 2.4, we see that $\left(z_{k}^{\alpha}+A\right)^{-1}$ exists and hence we have

$$
\tilde{V}(\zeta)=-\left(z_{k}^{\alpha}+A\right)^{-1}\left(\frac{1}{1-\zeta}-w_{0} \tilde{w}(\zeta)^{-1}\right) A u_{0},
$$

which implies that

$$
\begin{aligned}
V^{n} & =-\frac{1}{2 \pi i} \int_{|\zeta|=\rho} \zeta^{-n-1}\left(\frac{1}{1-\zeta}-w_{0} \tilde{w}(\zeta)^{-1}\right)\left(z_{k}^{\alpha}+A\right)^{-1} A u_{0} d \zeta \\
& =-\frac{1}{2 \pi i} \int_{|\zeta|=\rho} \zeta^{-n-1}\left(\frac{1}{1-\zeta}-w_{0} \tilde{w}(\zeta)^{-1}\right)\left(\frac{\delta(\zeta)}{k}\right) z_{k}^{-1}\left(z_{k}^{\alpha}+A\right)^{-1} A u_{0} d \zeta
\end{aligned}
$$

Let $\zeta=e^{-z k}, z=\frac{1}{k} \log \frac{1}{\rho}+i\left(-\frac{\theta}{k}\right),|\theta| \leq \pi$, we have

$$
V^{n}=\frac{1}{2 \pi i} \int_{\Gamma_{k}} e^{t_{n} z}\left(\frac{1}{1-\zeta}-w_{0} \tilde{w}(\zeta)^{-1}\right) \delta(\zeta) z_{k}^{-1}\left(z_{k}^{\alpha}+A\right)^{-1} A u_{0} d z,
$$

where $\Gamma_{k}=\{z \in \Gamma:|\Im z| \leq \pi / k\}$. For the details of the notation $\Gamma_{k}$, see the proof of [17, Lemma 3.2].

Denote

$$
\mu(\zeta)=\left(\frac{1}{1-\zeta}-w_{0} \tilde{w}(\zeta)^{-1}\right) \delta(\zeta)=\left(\frac{1}{1-\zeta}-w_{0} \tilde{w}(\zeta)^{-1}\right)(1-\zeta)^{1 / \alpha} \tilde{w}(\zeta)^{-1 / \alpha}
$$

we get

$$
V^{n}=-\frac{1}{2 \pi i} \int_{\Gamma_{k}} e^{t_{n} z} \mu(\zeta) z_{k}^{-1}\left(z_{k}^{\alpha}+A\right)^{-1} A u_{0} d z
$$

Thus, subtracting (2.13) from (2.10),

$$
\begin{aligned}
v\left(t_{n}\right)-V^{n}= & \frac{1}{2 \pi i} \int_{\Gamma_{k}} e^{t_{n} z}\left(\mu(\zeta) z_{k}^{-1}\left(z_{k}^{\alpha}+A\right)^{-1}-z^{-1}\left(z^{\alpha}+A\right)^{-1}\right) A u_{0} d z \\
& +\frac{1}{2 \pi i} \int_{\Gamma / \Gamma_{k}} e^{t_{n} z} z^{-1}\left(z^{\alpha}+A\right)^{-1} A u_{0} d z \\
= & I+I I .
\end{aligned}
$$

Further we denote

$$
K(z)=z^{-1}\left(z^{\alpha}+A\right)^{-1} A .
$$

For $I$, we have, by (2.19), with some suitable constant $c>0$,

$$
\begin{aligned}
\|I\| & \leq \frac{1}{2 \pi} \int_{\Gamma_{k}}\left|e^{t_{n} z}\right|\left|\mu(\zeta) K\left(z_{k}\right)-K(z)\right|\left\|u_{0}\right\||d z| \\
& \leq \frac{1}{2 \pi} \int_{\Gamma_{k}}\left|e^{t_{n} z}\right| C\left(k^{\alpha}|z|^{\alpha-1}+k\right)\left\|u_{0}\right\||d z| \\
& \leq C k^{\alpha} \int_{0}^{\infty} e^{-c t_{n} r}\left(r t_{n}\right)^{\alpha-1} t_{n}^{1-\alpha} d\left(r t_{n}\right) t_{n}^{-1}\left\|u_{0}\right\|+C k \int_{0}^{\infty} e^{-c t_{n} r} d\left(r t_{n}\right) t_{n}^{-1}\left\|u_{0}\right\| \\
& \leq C k^{\alpha} t_{n}^{-\alpha}\left\|u_{0}\right\|+C k t_{n}^{-1}\left\|u_{0}\right\| .
\end{aligned}
$$


For $I I$, we have, by (1.4) and noting that $\left(z^{\alpha}+A\right)^{-1} A=I-z^{\alpha}\left(z^{\alpha}+A\right)^{-1}$, with some suitable constant $c>0$,

$$
\begin{aligned}
\|I I\| & \leq \frac{1}{2 \pi} \int_{\Gamma / \Gamma_{k}}\left|e^{t_{n} z}\right|\left\|z^{-1}\left(z^{\alpha}+A\right)^{-1} A\right\||d z|\left\|u_{0}\right\| \leq C \int_{\Gamma / \Gamma_{k}} e^{-c t_{n}|z|}|z|^{-1}|d z|\left\|u_{0}\right\| \\
& \leq C k \int_{\frac{1}{k}}^{\infty} e^{-c t_{n} r} d r\left\|u_{0}\right\| \leq C k \int_{0}^{\infty} e^{-c t_{n} r} d\left(r t_{n}\right) t_{n}^{-1}\left\|u_{0}\right\| \leq C k t_{n}^{-1}\left\|u_{0}\right\| .
\end{aligned}
$$

The proof of Theorem 2.1 is now complete.

$\square$

LEMMA 2.6. Let $\zeta=e^{-z k}$ and $z \in \Gamma_{k}$. Let $\mu(\zeta), z_{k}$ and $K(z)$ be defined as in (2.12), (2.11), (2.15), respectively. We have

$$
\begin{aligned}
& \mu\left(e^{-z k}\right)-1=O\left((z k)^{\alpha}\right), \quad \text { as } z k \rightarrow 0 \\
& c|z| \leq\left|z_{k}\right| \leq C|z| \\
& \left\|K\left(z_{k}\right)-K(z)\right\| \leq C k|z|^{0} \\
& \left\|\mu(\zeta) K\left(z_{k}\right)-K(z)\right\| \leq C\left(k^{\alpha}|z|^{\alpha-1}+k\right) .
\end{aligned}
$$

Proof. We first show (2.16). It is sufficient to show

$$
\mu\left(e^{-w}\right)-1=O\left(w^{\alpha}\right), \quad \text { as } w \rightarrow 0 .
$$

By Lemma 2.5, we have

$$
\begin{aligned}
& \mu\left(e^{-w}\right)-1=\left(\frac{1}{1-e^{-w}}-w_{0}\left(\tilde{w}\left(e^{-w}\right)\right)^{-1}\right)\left(\left(1-e^{-w}\right) \tilde{w}(\zeta)^{-1}\right)^{1 / \alpha}-1 \\
& =\left(\frac{1-w_{0}\left(\tilde{w}\left(e^{-w}\right)\right)^{-1}\left(1-e^{-w}\right)}{1-e^{-w}}\right)\left(w^{\alpha}+c_{1} w^{1+\alpha}+c_{2} w^{1+2 \alpha}+\ldots\right)^{\frac{1}{\alpha}}-1
\end{aligned}
$$

and

$$
1-w_{0}\left(\tilde{w}\left(e^{-w}\right)\right)^{-1}\left(1-e^{-w}\right)=1+c_{1} w^{\alpha}+c_{2} w^{1+\alpha}+\ldots
$$

Hence

$$
\begin{aligned}
\mu\left(e^{-w}\right)-1= & \left(1+c_{1} w^{\alpha}+c_{2} w^{1+\alpha}+\ldots\right)\left(\frac{w}{1-e^{-w}}\right)\left(1+c_{1} w+c_{2} w^{1+\alpha}+\ldots\right)^{\frac{1}{\alpha}}-1 \\
= & \left(1+c_{1} w^{\alpha}+c_{2} w^{1+\alpha}+\ldots\right)\left(\frac{w}{w-\frac{w^{2}}{2}+\ldots}\right)\left(1+c_{1} w+c_{2} w^{1+\alpha}+\ldots\right)^{\frac{1}{\alpha}}-1 \\
= & \left(1+c_{1} w^{\alpha}+c_{2} w^{1+\alpha}+\ldots\right)\left(1+\frac{w}{2}+\ldots\right) \\
& \quad \cdot\left[1+\frac{1}{\alpha}\left(c_{1} w+c_{2} w^{1+\alpha}+\ldots\right)+\frac{\frac{1}{\alpha}\left(\frac{1}{\alpha}-1\right)}{2 !}\left(c_{1} w+c_{2} w^{1+\alpha}+\ldots\right)^{2}+\ldots\right]-1 \\
= & 1+O\left(w^{\alpha}\right)-1=O\left(w^{\alpha}\right), \quad \text { as } w \rightarrow 0,
\end{aligned}
$$

which shows (2.20). 
Next we show (2.17). Note that

$$
\frac{|z|}{\left|z_{k}\right|}=\frac{|z|}{\left|\frac{\delta\left(e^{-z k}\right)}{k}\right|}=\frac{|z k|}{\left|\delta\left(e^{-z k}\right)\right|} .
$$

To show (2.17), it suffices to prove $\frac{|z k|}{\left|\delta\left(e^{-z k}\right)\right|}$ has limit as $|z k| \rightarrow 0$, which follows from, noting that $\delta(\zeta)=(1-\zeta) \tilde{w}(\zeta)^{-1}$,

$$
\begin{aligned}
\lim _{w \rightarrow 0} \frac{w}{\delta\left(e^{-w}\right)} & =\lim _{w \rightarrow 0} \frac{w}{\left(\left(1-e^{-w}\right)\left(\tilde{w}\left(e^{-w}\right)\right)^{-1}\right)^{\frac{1}{\alpha}}}=\lim _{w \rightarrow 0} \frac{w}{\left(w^{\alpha}+c_{1} w^{1+\alpha}+c_{2} w^{1+2 \alpha}+\ldots\right)^{\frac{1}{\alpha}}} \\
& =\lim _{w \rightarrow 0} \frac{1}{\left(1+c_{1} w+c_{2} w^{1+\alpha}+\ldots\right)^{\frac{1}{\alpha}}}=1 .
\end{aligned}
$$

Hence we have proved, for any fixed constant $M>0$, there exists a constant $C$ such that

$$
\frac{|z|}{\left|z_{k}\right|} \leq C, \forall|z k| \leq M
$$

Similarly we may show $\frac{\left|z_{k}\right|}{|z|} \leq C, \forall|z k| \leq M$. Thus we get (2.17).

We now show (2.18). Note that

$$
\begin{aligned}
z_{k}-z & =\frac{\delta\left(e^{-z k}\right)}{k}-z=\frac{\delta\left(e^{-z k}\right)-z k}{k}=\frac{\left(\left(1-e^{-z k}\right)\left(\tilde{w}\left(e^{-z k}\right)\right)^{-1}\right)^{\frac{1}{\alpha}}-z k}{k} \\
& =\frac{\left((z k)^{\alpha}+c_{1}(z k)^{1+\alpha}+\ldots\right)^{\frac{1}{\alpha}}-z k}{k}=\frac{(z k)\left(1+c_{1}(z k)+\ldots\right)^{\frac{1}{\alpha}}-z k}{k} \\
& =\frac{(z k)\left(1+\frac{c_{2}}{\alpha}(z k)+\ldots\right)-z k}{k}=O\left(k z^{2}\right), \quad \text { as } k z \rightarrow 0 .
\end{aligned}
$$

Thus we have, following the proof of $[17,(4.6)]$ and noting $\left\|K^{\prime}(z)\right\| \leq C|z|^{-2}$ in $[17,(3.2)]$,

$$
\left\|K\left(z_{k}\right)-K(z)\right\| \leq C|z|^{-2} k|z|^{2}=C k .
$$

Finally we show (2.19). Following the same proof as in the proof of [17, Lemma 4.3], we have, noting that $\left|K\left(z_{k}\right)\right| \leq C|z|^{-1}$,

$$
\begin{aligned}
\left\|\mu(\zeta) K\left(z_{k}\right)-K(z)\right\| & \leq\left\|(\mu(\zeta)-1) K\left(z_{k}\right)\right\|+\left\|K\left(z_{k}\right)-K(z)\right\| \\
& \leq C|z k|^{\alpha}|z|^{-1}+k|z|^{0} \leq C k^{\alpha}|z|^{\alpha-1}+C k .
\end{aligned}
$$

Together these estimates complete the proof of Lemma 2.6.

2.2. A piecewise constant discontinuous Galerkin method with the convergence rate $O(k)$. We note that the convergence rate of the time stepping method (2.3) is only $O\left(k^{\alpha}\right), 0<\alpha<1$ with nonsmooth data. To derive a time stepping method for solving (1.5) with the convergence rate $O(k)$ for nonsmooth initial data $u_{0}$, we will approximate ${ }_{0}^{R} D_{t}^{1-\alpha} A u\left(t_{n}\right)$ by

$$
{ }_{0}^{R} D_{t}^{1-\alpha} A u\left(t_{n}\right) \approx k^{\alpha-1} \sum_{j=1}^{n} w_{n-j} A u\left(t_{j}\right),
$$


where we ignore the term $A u\left(t_{0}\right)$ in (2.1). More precisely, we choose $w_{n}=0$ in the summation $\sum_{j=0}^{n} w_{n-j} A u\left(t_{j}\right)$ in (2.1). It is easy to show that

$$
{ }_{0}^{R} D_{t}^{1-\alpha} A u\left(t_{n}\right)=k^{\alpha-1} \sum_{j=1}^{n} w_{n-j} A u\left(t_{j}\right)+O(k), \text { as } k \rightarrow 0 .
$$

To see this, by (2.1), it suffices to show that, for the fixed $t_{n}=n k=$ constant,

$$
k^{\alpha-1} w_{n}=t_{n}^{\alpha-1} O(k), \quad \text { as } k \rightarrow 0 .
$$

In fact, let $t_{n}$ be fixed, for example, assume that $t_{n}=1, n=1 / k$, we have, by (2.2),

$$
\begin{aligned}
\Gamma(1+\alpha) k^{\alpha-1} w_{n} & =k^{\alpha-1}\left(\alpha n^{\alpha-1}+(n-1)^{\alpha}-n^{\alpha}\right)=\alpha t_{n}^{\alpha-1}+(n-1)^{\alpha} k^{\alpha-1}-n^{\alpha} k^{\alpha-1} \\
& =t_{n}^{\alpha-1}\left(\alpha+\frac{(n-1)^{\alpha}}{n^{\alpha-1}}-\frac{n^{\alpha}}{n^{\alpha-1}}\right)=t_{n}^{\alpha-1}\left(\alpha+\frac{(1 / k-1)^{\alpha}}{(1 / k)^{\alpha-1}}-\frac{1}{k}\right) \\
& =t_{n}^{\alpha-1}\left(\alpha+\frac{(1-k)^{\alpha}-1}{k}\right)=t_{n}^{\alpha-1}\left(\alpha+\frac{\left(1-k \alpha+O\left(k^{2}\right)-1\right.}{k}\right) \\
& =t_{n}^{\alpha-1} O(k), \text { as } k \rightarrow 0,
\end{aligned}
$$

which implies (2.22) and therefore (2.21) follows.

Based on the approximation (2.21) for the Riemann-Liouville fractional derivative, we obtain the time stepping method (1.6) which was first introduced in McLean and Mustapha [19] for solving (1.5) by using the piecewise discontinuous Galerkin method.

TheOREM 2.7. Let the operator $A$ be a closed, densely defined linear operator satisfying (1.4). Let $u\left(t_{n}\right)$ and $U^{n}$ be the solutions of (1.5) and (1.6), respectively. Let $u_{0} \in L_{2}(\Omega)$. Then we have, with $0<\alpha<1$,

$$
\left\|u\left(t_{n}\right)-U^{n}\right\| \leq C k t_{n}^{-1}\left\|u_{0}\right\| .
$$

Proof. The proof is similar as the proof of Theorem 2.1. We shall use the same notations here as in the proof of Theorem 2.1.

Let $v(t)=u(t)-u_{0}$ and $V^{n}=U^{n}-u_{0}$. It suffices to show

$$
\left\|v\left(t_{n}\right)-V^{n}\right\| \leq C k t_{n}^{-1}\left\|u_{0}\right\|,
$$

which we will prove now.

This time $V^{n}, n=1,2,3, \ldots$ satisfy, by (1.6), with $V^{0}=0$,

$$
V^{n}-V^{n-1}+k^{\alpha} \sum_{j=1}^{n} w_{n-j} A V^{j}=-k^{\alpha} \sum_{j=1}^{n} w_{n-j} A u_{0}, n \geq 1 .
$$

Thus we have

$$
\sum_{n=1}^{\infty}\left(V^{n}-V^{n-1}\right) \zeta^{n}+k^{\alpha} \sum_{n=1}^{\infty} k^{\alpha}\left(\sum_{j=1}^{n} w_{n-j} A V^{j}\right) \zeta^{n}=-\sum_{n=1}^{\infty} k^{\alpha}\left(\sum_{j=1}^{n} w_{n-j} A u_{0}\right) \zeta^{n},
$$

which implies that

$$
(1-\zeta) \tilde{V}(\zeta)+k^{\alpha} \tilde{w}(\zeta) A \tilde{V}(\zeta)=-k^{\alpha}\left(\tilde{w}(\zeta) \frac{\zeta}{1-\zeta}\right) A u_{0}=-k^{\alpha}\left(\tilde{w}(\zeta) \frac{\zeta}{1-\zeta}\right) A u_{0}
$$


Denote

$$
\mu(\zeta)=\left(\frac{\zeta}{1-\zeta}\right) \delta(\zeta)=\left(\frac{\zeta}{1-\zeta}\right)(1-\zeta)^{1 / \alpha} \tilde{w}(\zeta)^{-1 / \alpha}
$$

we obtain

$$
V^{n}=-\frac{1}{2 \pi i} \int_{\Gamma_{k}} e^{t_{n} z} \mu(\zeta) z_{k}^{-1}\left(z_{k}^{\alpha}+A\right)^{-1} A u_{0} d z
$$

The rest of the proof is to bound $\left\|v\left(t_{n}\right)-V^{n}\right\|$ which can be done by using (2.27) below and the arguments for estimating (2.14) in the proof of Theorem 2.1. We omit the details here.

$\square$

Lemma 2.8. Let $\zeta=e^{-z k}$ and $z \in \Gamma_{k}$. Let $\mu(\zeta)$ and $K(z)$ be defined as in (2.24) and (2.15), respectively. We have

$$
\begin{aligned}
& \mu\left(e^{-z k}\right)-1=O(z k), \quad \text { as } z k \rightarrow 0, \\
& \left\|\mu(\zeta) K\left(z_{k}\right)-K(z)\right\| \leq C k|z|^{0} .
\end{aligned}
$$

Proof. We first show (2.26). It is sufficient to show

$$
\mu\left(e^{-w}\right)-1=O(w), \quad \text { as } w \rightarrow 0,
$$

which follows from, by Lemma 2.5,

$$
\begin{aligned}
& \mu\left(e^{-w}\right)-1=\left(\frac{e^{-w}}{1-e^{-w}}\right)\left(\left(1-e^{-w}\right) \tilde{w}(\zeta)^{-1}\right)^{1 / \alpha}-1 \\
& =e^{-w}\left(\frac{w}{1-e^{-w}}\right)\left(1+c_{1} w+c_{2} w^{1+\alpha}+\ldots\right)-1=O(w), \text { as } w \rightarrow 0 .
\end{aligned}
$$

We next show (2.27). Following the same proof as in the proof of [17, Lemma 4.3], we have, by (2.18), (2.26) and noting again that $\left|K\left(z_{k}\right)\right| \leq C|z|^{-1}$,

$$
\begin{aligned}
\left\|\mu(\zeta) K\left(z_{k}\right)-K(z)\right\| & \leq\left\|(\mu(\zeta)-1) K\left(z_{k}\right)\right\|+\left\|K\left(z_{k}\right)-K(z)\right\| \\
& \leq C|z k \| z|^{-1}+k|z|^{0} \leq C k .
\end{aligned}
$$

Together these estimates complete the proof of Lemma 2.8.

2.3. A new time discretization with the convergence rate $O\left(k^{1+\alpha}\right), 0<$ $\alpha<1$. In this subsection, we shall introduce a new time discretization scheme for solving (1.5) by using the convolution quadrature method. We prove that this method has the convergence rate $O\left(k^{1+\alpha}\right)$ with nonsmooth initial data $u_{0}$.

Following the idea in Lubich et al. [17], we shall approximate the time derivative $u_{t}\left(t_{n}\right)$ by using a second order backward difference method

$$
u_{t}\left(t_{n}\right)=\frac{\frac{3}{2} u\left(t_{n}\right)-2 u\left(t_{n-1}\right)+\frac{1}{2} u\left(t_{n-2}\right)}{k}+O\left(k^{2}\right), \quad \text { as } k \rightarrow 0 .
$$

We define the following finite difference method for solving (1.5), with $U^{n} \approx u\left(t_{n}\right)$ 
and $c_{0}=1 / 2$,

$$
\begin{aligned}
& \bar{D} U^{n}+k^{\alpha-1}\left(\sum_{j=1}^{n} w_{n-j} A U^{j}+c_{0} w_{n-1} A u_{0}\right)=0, n \geq 2 \\
& \bar{D} U^{n}+k^{\alpha-1}\left(\sum_{j=1}^{n} w_{n-j} A U^{j}+c_{0} w_{n-1} A u_{0}\right)=0, n=1 \\
& U^{0}=u_{0}, U^{-1}=u_{0}
\end{aligned}
$$

where

$$
\bar{D} U^{n}=\frac{\frac{3}{2} U^{n}-2 U^{n-1}+\frac{1}{2} U^{n-2}}{k}, n \geq 1,
$$

and $w_{j}, j=0,1,2, \ldots, n-1$ are defined by (1.7). Here we use a modification term $c_{0} w_{n-1} A u_{0}$ as in Lubich et al. [17, (1.18)].

TheOREM 2.9. Let the operator $A$ be a closed, densely defined linear operator satisfying (1.4). Let $u\left(t_{n}\right)$ and $U^{n}$ be the solutions of (1.5) and (2.29)-(2.31), respectively. Let $u_{0} \in L_{2}(\Omega)$. Then we have, with $0<\alpha<1$,

$$
\left\|u\left(t_{n}\right)-U^{n}\right\| \leq C k^{1+\alpha} t_{n}^{-1-\alpha}\left\|u_{0}\right\| .
$$

To prove Theorem 2.9, we need to show that $z_{k}^{\alpha} \in \Sigma_{\theta}$ for some $\theta \in(\pi / 2, \pi)$ where $z_{k}$ is defined in (2.36) below.

Lemma 2.10. Let $\theta>\pi / 2$ be close to $\pi / 2$. Let $z \in \Gamma_{k}$ with $\Gamma_{k}=\{z: z \in$ $\Gamma,|\Im z| \leq \pi / k\}$ where $\Gamma=\{z:|\arg z|=\theta\}$ (with $\Im z$ running from $-\infty$ to $\infty$ ). Let $z_{k}=\delta(\zeta) / k$ with $\zeta=e^{-z k}$ be defined by (2.36), where

$$
\delta(\zeta)^{\alpha}=\left(\frac{3}{2}-2 \zeta+\frac{1}{2} \zeta^{2}\right) \tilde{w}(\zeta)^{-1},
$$

and $\tilde{w}(\zeta)=\sum_{j=0}^{\infty} w_{j} \zeta^{j}$ with $w_{j}, j=0,1,2, \ldots$ defined by (2.5). Then there exists $\theta_{0} \in(\pi / 2, \pi)$ such that

$$
z_{k}^{\alpha} \in \Sigma_{\theta_{0}}, \quad \text { for all } z \in \Gamma_{\theta} \text {. }
$$

Proof. The proof is similar as the proof of Lemma 2.4. We omit the proof here. $\square$

Proof. [Proof of Theorem 2.9] Let $v(t)=u(t)-u_{0}$ and $V^{n}=U^{n}-u_{0}$. It suffices to show

$$
\left\|v\left(t_{n}\right)-V^{n}\right\| \leq C k^{1+\alpha} t_{n}^{-1-\alpha}\left\|u_{0}\right\|,
$$

which we will prove now.

This time $V^{n}, n=1,2,3, \ldots$ satisfy, by (2.29)-(2.31), with $c_{0}=1 / 2$,

$$
\begin{aligned}
& \left(\frac{3}{2} V^{n}-2 V^{n-1}+\frac{1}{2} V^{n-2}\right)+k^{\alpha}\left(\sum_{j=1}^{n} w_{n-j} A V^{j}+c_{0} w_{n-1} A u_{0}\right) \\
& \quad=-k^{\alpha}\left(\sum_{j=1}^{n} w_{n-j} A u_{0}+c_{0} w_{n-1} A u_{0}\right), n \geq 1, \\
& V^{0}=0, V^{-1}=0 .
\end{aligned}
$$


Thus we have

$$
\begin{aligned}
\sum_{n=1}^{\infty}\left(\frac{3}{2} V^{n}-2 V^{n-1}+\frac{1}{2} V^{n-2}\right) \zeta^{n} & +\sum_{n=1}^{\infty} k^{\alpha}\left(\sum_{j=1}^{n} w_{n-j} A V^{j}+c_{0} w_{n-1} A u_{0}\right) \zeta^{n} \\
& =-\sum_{n=1}^{\infty} k^{\alpha}\left(\sum_{j=1}^{n} w_{n-j} A u_{0}+c_{0} w_{n-1} A u_{0}\right) \zeta^{n}
\end{aligned}
$$

which implies that

$$
\begin{aligned}
\left(\frac{3}{2}-2 \zeta+\frac{1}{2} \zeta^{2}\right) \tilde{V}(\zeta)+k^{\alpha} \tilde{w}(\zeta) A \tilde{V}(\zeta) & =-k^{\alpha} \tilde{w}(\zeta)\left(\left(1+c_{0}\right) \zeta+\zeta^{2}+\zeta^{3}+\ldots\right) A u_{0} \\
& =-k^{\alpha} \tilde{w}(\zeta)\left(\frac{\zeta}{1-\zeta}+c_{0} \zeta\right) A u_{0}
\end{aligned}
$$

Hence

$$
\left(\frac{\delta(\zeta)}{k}\right)^{\alpha} \tilde{V}(\zeta)+A \tilde{V}(\zeta)=-\left(\frac{\zeta}{1-\zeta}+c_{0} \zeta\right) A u_{0}
$$

where the generating function $\delta(\zeta)^{\alpha}$ is defined by (2.33), see also [17].

Denote

$$
z_{k}=\frac{\delta(\zeta)}{k}
$$

By Lemma 2.10, we see that $\left(z_{k}^{\alpha}+A\right)^{-1}$ exists and hence we have

$$
\tilde{V}(\zeta)=-\left(z_{k}^{\alpha}+A\right)^{-1}\left(\frac{\zeta}{1-\zeta}+c_{0} \zeta\right) A u_{0}
$$

Denote

$$
\mu(\zeta)=\left(\frac{\zeta}{1-\zeta}+c_{0} \zeta\right) \delta(\zeta)=\left(\frac{\zeta}{1-\zeta}+c_{0} \zeta\right)\left(\frac{3}{2}-2 \zeta+\frac{1}{2} \zeta^{2}\right)^{1 / \alpha} \tilde{w}(\zeta)^{-1 / \alpha}
$$

we get

$$
V^{n}=-\frac{1}{2 \pi i} \int_{\Gamma_{k}} e^{t_{n} z} \mu(\zeta) z_{k}^{-1}\left(z_{k}^{\alpha}+A\right)^{-1} A u_{0} d z
$$

The rest of the proof is to bound $\left\|v\left(t_{n}\right)-V^{n}\right\|$ which can be done by using (2.42) below and the arguments for estimating (2.14) in the proof of Theorem 2.1. We omit the details here.

口

Lemma 2.11. Let $w_{j}, j=0,1,2, \ldots, n, \ldots$ be defined as in (2.5). Then we have the following singularity expansion, with $\zeta=e^{-z k}$,

$$
\left(\frac{3}{2}-2 \zeta-\frac{1}{2} \zeta^{2}\right) \tilde{w}(\zeta)^{-1}=(z k)^{\alpha}+c_{1}(z k)^{1+2 \alpha}+c_{2}(z k)^{2+\alpha} \ldots
$$

for some suitable constants $c_{1}, c_{2}, \ldots$. 
Proof. We have, by the expansion of $\tilde{w}(z)$ in $(2.8)$,

$$
\begin{aligned}
& \left(\frac{3}{2}-2 \zeta-\frac{1}{2} \zeta^{2}\right) \tilde{w}(\zeta)^{-1}=\left(\frac{3}{2}-2 e^{-z k}-\frac{1}{2} e^{-2 z k}\right)\left(\tilde{w}\left(e^{-z k}\right)\right)^{-1} \\
& =\left((z k)-\frac{(z k)^{3}}{3}++\ldots\right)\left((z k)^{1-\alpha}+c_{1}(z k)^{2}+c_{2}(z k)^{3-\alpha}+\ldots\right)^{-1} \\
& =(z k)^{\alpha}+c_{1}(z k)^{1+2 \alpha}+c_{2}(z k)^{2+\alpha} \ldots
\end{aligned}
$$

Together these estimates complete the proof of Lemma 2.11. $\square$

Lemma 2.12. Let $\zeta=e^{-z k}$ and $z \in \Gamma_{k}$. Let $\mu(\zeta)$ and $z_{k}$ be defined as in (2.37) and (2.36), respectively. We have

$$
\begin{aligned}
& \mu\left(e^{-z k}\right)-1=O\left((z k)^{1+\alpha}\right), \quad \text { as } z k \rightarrow 0, \\
& c|z| \leq\left|z_{k}\right| \leq C|z|, \\
& \left\|K\left(z_{k}\right)-K(z)\right\| \leq C k^{1+\alpha}|z|^{\alpha}, \\
& \left\|\mu(\zeta) K\left(z_{k}\right)-K(z)\right\| \leq C k^{1+\alpha}|z|^{\alpha} .
\end{aligned}
$$

Proof. We first show (2.39). It is sufficient to show

$$
\mu\left(e^{-w}\right)-1=O\left(w^{1+\alpha}\right), \quad \text { as } w \rightarrow 0,
$$

which follows from, by Lemma 2.11,

$$
\begin{aligned}
& \mu\left(e^{-w}\right)-1=\left(\frac{e^{-w}}{1-e^{-w}}+c_{0} e^{-w}\right)\left(\left(\frac{3}{2}-2 e^{-w}-e^{-2 w}\right) \tilde{w}(\zeta)^{-1}\right)^{1 / \alpha}-1 \\
& =\left(\frac{e^{-w}}{1-e^{-w}}+c_{0} e^{-w}\right)\left(w^{\alpha}+c_{1} w^{1+2 \alpha}+c_{2} w^{2+\alpha}+\ldots\right)^{\frac{1}{\alpha}}-1=O\left(w^{1+\alpha}\right), \text { as } w \rightarrow 0 .
\end{aligned}
$$

Next we show (2.40). Note that

$$
\frac{|z|}{\left|z_{k}\right|}=\frac{|z|}{\left|\frac{\delta\left(e^{-z k}\right)}{k}\right|}=\frac{|z k|}{\left|\delta\left(e^{-z k}\right)\right|} .
$$

To show (2.40), it suffices to prove $\frac{|z k|}{\left|\delta\left(e^{-z k}\right)\right|}$ has limit as $|z k| \rightarrow 0$, which follows from, noting that $\delta(\zeta)=\left(\frac{3}{2}-2 \zeta-\frac{1}{2} \zeta^{2}\right) \tilde{w}(\zeta)^{-1}$,

$$
\begin{aligned}
\lim _{w \rightarrow 0} \frac{w}{\delta\left(e^{-w}\right)} & =\lim _{w \rightarrow 0} \frac{w}{\left(\left(\frac{3}{2}-2 e^{-w}-\frac{1}{2} e^{-2 w}\right) \tilde{w}\left(e^{-w}\right)^{-1}\right)^{\frac{1}{\alpha}}} \\
& =\lim _{w \rightarrow 0} \frac{w}{\left(w^{\alpha}+c_{1} w^{1+2 \alpha}+c_{2} w^{2+\alpha}+\ldots\right)^{\frac{1}{\alpha}}} \\
& =\lim _{w \rightarrow 0} \frac{1}{\left(1+c_{1} w^{1+\alpha}+c_{2} w^{2}+\ldots\right)^{\frac{1}{\alpha}}}=1 .
\end{aligned}
$$

Hence we have proved, for any fixed constant $M>0$, there exists a constant $C$ such that

$$
\frac{|z|}{\left|z_{k}\right|} \leq C, \forall|z k| \leq M
$$


Similarly we may show $\frac{\left|z_{k}\right|}{|z|} \leq C, \forall|z k| \leq M$. Thus we get (2.40).

We now show (2.41). Note that

$$
\begin{aligned}
z_{k}-z & =\frac{\left(\left(\frac{3}{2}-2 e^{-z k}-\frac{1}{2} e^{-2 z k}\right) \tilde{w}\left(e^{-z k}\right)^{-1}\right)^{\frac{1}{\alpha}}-z k}{k} \\
& =\frac{\left((z k)^{\alpha}+c_{1}(z k)^{1+2 \alpha}+c_{2}(z k)^{2+\alpha}+\ldots\right)^{\frac{1}{\alpha}}-z k}{k} \\
& =\frac{(z k)\left(1+c_{1}(z k)^{1+\alpha}+c_{2}(z k)+\ldots\right)^{\frac{1}{\alpha}}-z k}{k} \\
& =\frac{(z k)\left(1+c_{1}(z k)^{1+\alpha}+c_{2}(z k)+\ldots\right)-z k}{k}=O\left(k^{1+\alpha} z^{2+\alpha}\right), \quad \text { as } z k \rightarrow 0 .
\end{aligned}
$$

Thus we have, following the proof of $[17,(4.6)]$ and noting $\left\|K^{\prime}(z)\right\| \leq C|z|^{-2}$ in $[17,(3.12)]$,

$$
\left\|K\left(z_{k}\right)-K(z)\right\| \leq C|z|^{-2} k^{1+\alpha}|z|^{2+\alpha}=C k^{1+\alpha}|z|^{\alpha} .
$$

Finally we show (2.42). Following the same proof as in the proof of [17, Lemma 4.3], we have, noting that $\left|K\left(z_{k}\right)\right| \leq C|z|^{-1}$,

$$
\begin{aligned}
\left\|\mu(\zeta) K\left(z_{k}\right)-K(z)\right\| & \leq\left\|(\mu(\zeta)-1) K\left(z_{k}\right)\right\|+\left\|K\left(z_{k}\right)-K(z)\right\| \\
& \leq C|z k|^{1+\alpha}|z|^{-1}+k^{1+\alpha}|z|^{\alpha} \leq C k^{1+\alpha}|z|^{\alpha} .
\end{aligned}
$$

Together these estimates complete the proof of Lemma 2.12. $\square$

REMARK 2.13. We remark that assuming that $u_{0} \in \mathcal{D}(A)$ rather than $u_{0} \in L_{2}(\Omega)$ reduces the singular behavior of the error bound at $t=0$. We can also prove the convergence rates $O\left(k^{r}\right)$ with $r=\alpha, 1$ and $1+\alpha$ for $0<\alpha<1$, respectively as in the Theorems 2.1, 2.7, 2.9, see Lubich et al. [17, p.16]

3. Inhomogeneous problem. In this section we will consider the time stepping method for solving the inhomogeneous problem (1.1) based on the time stepping method introduced in Section 2 for the homogeneous problem.

Let $u(t)-u_{0}=v(t)$. Then (1.1) is equivalent to

$$
{ }_{0}^{C} D_{t}^{\alpha} v(t)+A v(t)=-A u_{0}+f(t), 0<t \leq T, \quad \text { with } v(0)=0 .
$$

With $V^{n} \approx v\left(t_{n}\right), n=0,1,2, \ldots, N$, we define the following time stepping method for solving (3.1), with $V^{0}=0$ and $c_{0}=1 / 2$,

$$
\begin{aligned}
& k^{-\alpha} \sum_{j=1}^{n} \delta_{n-j}^{(\alpha)} V^{j}+A V^{j}=-A u_{0}+f\left(t_{n}\right)+c_{0}\left(-A u_{0}+f(0)\right), n=1, \\
& k^{-\alpha} \sum_{j=1}^{n} \delta_{n-j}^{(\alpha)} V^{j}+A V^{j}=-A u_{0}+f\left(t_{n}\right), n=2,3, \ldots, N,
\end{aligned}
$$

where $\delta_{j}^{(\alpha)}, j=0,1,2, \ldots$ are generated by $\delta(\zeta)^{\alpha}=\sum_{j=0}^{\infty} \delta_{j}^{(\alpha)} \zeta^{j}$. Here $\delta(\zeta)$ is defined by $(2.33)$.

THEOREM 3.1. Let the operator $A$ be a closed, densely defined linear operator satisfying (1.4). Let $u\left(t_{n}\right)$ and $U^{n}$ be the solutions of (3.1) and (3.2)-(3.3), respectively. Let $u_{0} \in L_{2}(\Omega)$ and $f \in H^{2}\left(0, T ; L_{2}(\Omega)\right)$. Then we have, with $0<\alpha<1$, 


$$
\left\|u\left(t_{n}\right)-U^{n}\right\| \leq C k^{1+\alpha}\left(t_{n}^{-1-\alpha}\left\|u_{0}\right\|+t_{n}^{-1}\|f(0)\|+\left\|f^{\prime}(0)\right\|+\int_{0}^{t_{n}}\left\|f^{\prime \prime}(s)\right\|_{L_{2}(\Omega)}\right) .
$$

To prove Theorem 3.1, we need the following lemma.

Lemma 3.2. Let $z_{k}$ be defined as in (2.36). We have

$$
\left\|\left(z^{\alpha}+A\right)^{-1} z^{-2}-\left(z_{k}^{\alpha}+A\right)^{-1}\left(k \sum_{n=1}^{\infty} t_{n} \zeta^{n}\right)\right\| \leq C k^{1+\alpha}|z|^{-1} .
$$

Proof. We have

$$
\begin{aligned}
& \left\|\left(z^{\alpha}+A\right)^{-1} z^{-2}-\left(z_{k}^{\alpha}+A\right)^{-1}\left(k \sum_{n=1}^{\infty} t_{n} \zeta^{n}\right)\right\| \\
& \leq\left\|\left(z^{\alpha}+A\right)^{-1} z^{-2}-\left(z_{k}^{\alpha}+A\right)^{-1} z_{k}^{-2}\right\|+\left\|\left(z_{k}^{\alpha}+A\right)^{-1} z_{k}^{-2}\left(1-z_{k}^{2} k \sum_{n=1}^{\infty} t_{n} \zeta^{n}\right)\right\| .
\end{aligned}
$$

It is easy to show that

$$
\left\|1-z_{k}^{2} k \sum_{n=1}^{\infty} t_{n} \zeta^{n}\right\| \leq C|z k|^{1+\alpha} .
$$

The rest of the proof of Lemma 3.2 follows from the arguments in the proof of (2.27).

Proof. [Proof of Theorem 3.1] The proof is similar to the arguments in [11] and [12] for the error estimates of the inhomogeneous problem.

Denote

$$
f(t)=f(0)+R(t), \quad R(t)=t f^{\prime}(0)+\left(t * f^{\prime \prime}\right)(t) .
$$

Here $f * g$ denotes the convolution of $f$ and $g$.

Taking the Laplace transform in (3.1), we have

$$
z^{\alpha} \hat{v}(z)+A \hat{v}(z)=-A u_{0} z^{-1}+\hat{f}(z)=-A u_{0} z^{-1}+f(0) z^{-1}+\hat{R}(z),
$$

which implies that

$$
v(t)=\frac{1}{2 \pi i} \int_{\Gamma} e^{z t}\left(\left(z^{\alpha}+A\right)^{-1} z^{-1}\left(-A u_{0}+f(0)\right)+\left(z^{\alpha}+A\right)^{-1} \hat{R}(z)\right) d z .
$$

Taking the discrete Laplace transform in (3.2)-(3.3), we have

$$
\begin{aligned}
& \sum_{n=1}^{\infty}\left(k^{-\alpha} \sum_{j=1}^{n} \delta_{n-j}^{(\alpha)} V^{j}\right) \zeta^{n}+\sum_{n=1}^{\infty}\left(A V^{n}\right) \zeta^{n} \\
& =\sum_{n=1}^{\infty}\left(-A u_{0}+f(0)\right) \zeta^{n}+\sum_{n=1}^{\infty} R\left(t_{n}\right) \zeta^{n}+c_{0}\left(-A u_{0}+f(0)\right) \zeta
\end{aligned}
$$


which implies that

$$
\begin{aligned}
V^{n} & =\frac{1}{2 \pi i} \int_{\Gamma_{k}} e^{z t_{n}}\left(z_{k}^{\alpha}+A\right)^{-1} z_{k}^{-1} \mu\left(e^{-z k}\right)\left(-A u_{0}+f(0)\right) d z \\
& +\frac{1}{2 \pi i} \int_{\Gamma_{k}} e^{z t_{n}}\left(z_{k}^{\alpha}+A\right)^{-1} k\left(\sum_{n=1}^{\infty} R\left(t_{n}\right) \zeta^{n}\right) d z
\end{aligned}
$$

where $\mu(\zeta)$ and $z_{k}$ are defined by (2.37) and (2.36), respectively.

The rest of the proof may be completed by using Lemma 3.2 and the arguments in Jin et al. [11], [12].

Together these estimates complete the proof of Theorem 3.1.

4. Numerical examples. In this section, we will consider the numerical simulations of the different time discretization schemes discussed in Section 2 for solving (1.5). We only consider the homogeneous problem and illustrate the experimentally determined convergence rates with nonsmooth data. Similarly we may illustrate the inhomogeneous problem with some sufficiently smooth source term $f$.

Let us consider the following time fractional partial differential equation in one dimensional case.

$$
\begin{aligned}
& { }_{0}^{C} D_{t}^{\alpha} u(x, t)-u_{x x}=0, \quad 0<x<1, \quad 0<t \leq T, \\
& u(0, t)=u(1, t)=0, \\
& u(x, 0)=u_{0}(x) .
\end{aligned}
$$

Let $0<t_{0}<t_{1}<\cdots<t_{N}=T$ be the time partition of $[0, T]$ with $T=1$ and $k$ the time step size. Let $N_{h}$ be a positive integer. Let $0=x_{0}<x_{1}<x_{2}<\cdots<x_{N_{h}}=1$ be the space partition and $h$ the space step size. The space is discretized by using the standard linear finite element method.

\begin{tabular}{cccccc}
\hline$\alpha$ & $k=2^{-4}$ & $k=2^{-5}$ & $k=2^{-6}$ & $k=2^{-7}$ & $k=2^{-8}$ \\
\hline 0.1 & $1.57 \mathrm{e}-01$ & $1.27 \mathrm{e}-01$ & $9.84 \mathrm{e}-02$ & $7.13 \mathrm{e}-02$ & $4.59 \mathrm{e}-02$ \\
& 0.308 & 0.370 & 0.464 & 0.635 & \\
0.3 & $1.64 \mathrm{e}-01$ & $1.21 \mathrm{e}-01$ & $8.62 \mathrm{e}-02$ & $5.76 \mathrm{e}-02$ & $3.43 \mathrm{e}-02$ \\
& 0.434 & 0.492 & 0.581 & 0.747 & \\
0.8 & $1.90 \mathrm{e}-02$ & $1.06 \mathrm{e}-02$ & $5.75 \mathrm{e}-03$ & $2.99 \mathrm{e}-03$ & $1.42 \mathrm{e}-03$ \\
& 0.848 & 0.877 & 0.939 & 1.076 & \\
0.9 & $7.73 \mathrm{e}-03$ & $3.99 \mathrm{e}-03$ & $2.04 \mathrm{e}-03$ & $1.00 \mathrm{e}-03$ & $4.52 \mathrm{e}-04$ \\
& 0.953 & 0.970 & 1.023 & 1.151 & \\
\hline
\end{tabular}

TABLE 1

Time convergence rates with the different $\alpha \in(0,1)$ for the numerical method (2.3)

We first consider the scheme (2.3) and the convergence rate was proved to be $O\left(k^{\alpha}\right)$ for both smooth and nonsmooth data in Theorem 2.1. To observe this convergence rate, we first calculate the reference solution uref $(t)$ at $T=1$ with $h_{\text {ref }}=2^{-6}$ and $k_{\text {ref }}=2^{-10}$. We then use $h=2^{-6}$ and $k=k a p p a * k_{\text {ref }}$ with kappa $=$ $\left[2^{2}, 2^{3}, 2^{4}, 2^{5}, 2^{6}\right]$ to obtain the approximate solution at $u(T)$ with $T=1$. Let $e_{k}$ 
denote the error of $u(T)$ at $T=1$ with the time step size $k$ and the fixed space step size $h=2^{-6}$. By Theorem 2.1, we have

$$
\left\|e_{k}\right\| \leq C k^{\alpha}
$$

Thus the convergence rate $\alpha$ is determined experimentally by

$$
\alpha \approx \log 2\left(\frac{\left\|e_{2 k}\right\|}{\left\|e_{k}\right\|}\right)
$$

Choosing the nonsmooth initial data $u_{0}=\chi_{[0,1 / 2]}$, we observe, in Table 1 , that the experimentally determined convergence rate is indeed almost $O\left(k^{\alpha}\right)$ for the different $\alpha \in(0,1)$ with the nonsmooth initial data.

We next consider the numerical method (1.6) proposed by McLean and Mustapha [18] which has the convergence rate $O(k)$ for both smooth and nonsmooth initial data. Using the same notations and the same initial data as in Table 1, we found, in Table 2, that the experimentally determined convergence rate of this method is indeed approximately 1 .

\begin{tabular}{cccccc}
\hline$\alpha$ & $k=2^{-4}$ & $k=2^{-5}$ & $k=2^{-6}$ & $k=2^{-7}$ & $k=2^{-8}$ \\
\hline 0.1 & $1.20 \mathrm{e}-04$ & $6.53 \mathrm{e}-05$ & $3.43 \mathrm{e}-05$ & $1.71 \mathrm{e}-05$ & $7.73 \mathrm{e}-06$ \\
& 0.876 & 0.929 & 1.01 & 1.14 & \\
0.3 & $6.99 \mathrm{e}-04$ & $3.57 \mathrm{e}-04$ & $1.77 \mathrm{e}-04$ & $8.46 \mathrm{e}-05$ & $3.69 \mathrm{e}-05$ \\
& 0.972 & 1.01 & 1.07 & 1.19 & \\
0.8 & $1.29 \mathrm{e}-03$ & $6.01 \mathrm{e}-04$ & $2.83 \mathrm{e}-04$ & $1.30 \mathrm{e}-04$ & $5.54 \mathrm{e}-05$ \\
& 1.103 & 1.088 & 1.120 & 1.233 & \\
0.9 & $9.66 \mathrm{e}-04$ & $4.35 \mathrm{e}-04$ & $2.02 \mathrm{e}-04$ & $9.22 \mathrm{e}-05$ & $3.91 \mathrm{e}-05$ \\
& 1.151 & 1.109 & 1.130 & 1.238 & \\
\hline
\end{tabular}

TABLE 2

Time convergence rates with the different $\alpha \in(0,1)$ for the numerical method (1.6)

In Figure 1, by using the time discretization method (1.6), we show how the error varies with $t_{n}=0.01,0.1,0.2,0.4,0.6,0.8,1.0$ by choosing $\alpha=0.3$ and the time step size $k=2^{-6}$ and the space step size $h=2^{-6}$. Here the reference solution is calculated by using $k_{\text {ref }}=2^{-10}$ and $h_{\text {ref }}=2^{-6}$.

Finally we consider the improved numerical method (2.29)-(2.31) which has the convergence rate $O\left(k^{1+\alpha}\right)$ for both smooth and nonsmooth data. Using the same notations and the same initial data as in Tables 1 and 2, we found, in Table 3, that the experimentally determined convergence rate is approximately $k^{1+\alpha}$ ( actually the experimentally determined convergence rate is better than $1+\alpha$ ) as we expected.

Acknowledgments. The second author thanks the organizers of the Mini-Symposium: "Numerical Methods for Fractional Differential Equations "on the conference for the Mathematics of Finite Elements and Applications (MAFELAP), 2016 in Brunel, UK. Some results in this paper were presented in that Mini-Symposium. 


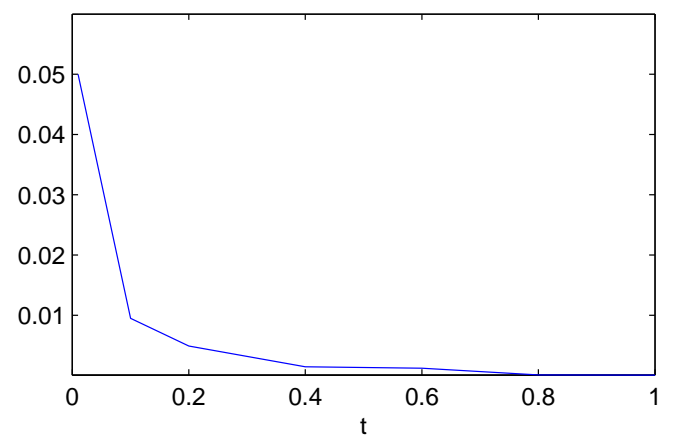

FIG. 1. The error $\left\|U^{n}-u\left(t_{n}\right)\right\|$ as a function of $t_{n}$

\begin{tabular}{cccccc}
\hline$\alpha$ & $k=2^{-4}$ & $k=2^{-5}$ & $k=2^{-6}$ & $k=2^{-7}$ & $k=2^{-8}$ \\
\hline 0.1 & $2.08 \mathrm{e}-04$ & $9.34 \mathrm{e}-05$ & $4.17 \mathrm{e}-05$ & $1.82 \mathrm{e}-05$ & $7.35 \mathrm{e}-06$ \\
& 1.159 & 1.162 & 1.198 & 1.307 & \\
0.3 & $2.16 \mathrm{e}-04$ & $7.77 \mathrm{e}-05$ & $2.88 \mathrm{e}-05$ & $1.07 \mathrm{e}-05$ & $3.78 \mathrm{e}-06$ \\
& 1.472 & 1.431 & 1.428 & 1.502 & \\
0.8 & $1.04 \mathrm{e}-04$ & $2.25 \mathrm{e}-05$ & $4.95 \mathrm{e}-06$ & $1.08 \mathrm{e}-06$ & $2.22 \mathrm{e}-07$ \\
& 2.199 & 2.187 & 2.203 & 2.273 & \\
0.9 & $9.87 \mathrm{e}-05$ & $2.27 \mathrm{e}-05$ & $5.26 \mathrm{e}-06$ & $1.23 \mathrm{e}-06$ & $2.80 \mathrm{e}-07$ \\
& 2.122 & 2.107 & 2.096 & 2.134 & \\
\hline
\end{tabular}

TABLE 3

Time convergence rates with the different $\alpha \in(0,1)$ for the numerical methods (2.29)-(2.31)

[1] C.-M. Chen, F. Liu, V. Anh, and I. Turner Numerical methods for solving a two-dimensional variable-order anomalous sub-diffusion equation, Math. Comp., 81(2012), pp. 345-366.

[2] E. Cuesta, Ch. Lubich, and C. Palencia, Convolution quadrature time discretization of fractional diffusion-wave equations, Math. Comp., 75(2006), pp. 673-696.

[3] M. Cui, Compact finite difference method for the fractional diffusion equation, J. Comput. Phys., 228(2009), pp. 7792-7804.

[4] W. Deng, Finite element method for the space and time fractional Fokker-Planck equation, SIAM J. Numer. Anal, 47(2008), pp. 204-226.

[5] K. Diethelm, An algorithm for the numerical solution of differential equations of fractional order, Electronic Transactions on Numerical Analysis, 5(1997), pp. 1-6.

[6] P. Flajolet, Singularity analysis and asymptotics of Bernoulli sums, Theoret. Comput. Sci., 215(1999), pp. 371-381.

[7] N. J. Ford, J. Xiao, and Y. Yan, A finite element method for time-fractional partial differential equations, Fract. Calc. Appl. Anal., 14(2011), pp. 454-474.

[8] G.-H. Gao, Z.-Z. Sun, and H.-W. Zhang, A new fractional numerical differentiation formula to approximate the Caputo fractional derivative and its applications, J. Comput. Phys., 259(2014), pp. 33-50.

[9] B. Jin, R. Lazarov, and Z. Zhou, Error estimates for a semidiscrete finite element method for fractional order parabolic equations, SIAM J. Numer. Anal., 51(2013), pp. 445-466.

[10] B. Jin, R. Lazarov, and Z. Zhou, An analysis of the L1 scheme for the subdiffusion equation with nonsmooth data, IMA J. of Numer. Anal., 36(2016), pp. 197-221.

[11] B. Jin, B. Li, and Z. Zhou, An analysis of the Crank-Nikolson method for subdiffusion, IMA J. Numer. Anal., in press, (2017), arXiv:1607.06948.

[12] B. Jin, B. Li, and Z. Zhou, Correction of higher-order BDF convolution quadrature for fractional evolution equations, (2017), arXiv:1703.08808. 
[13] T. A. M. Langlands and B. I. Henry, The accuracy and stability of an implicit solution method for the fractional diffusion equation, J. Comput. Phys., 205(2005), pp. 719-736.

[14] C. Li and H. Ding, Higher order finite difference method for the reaction and anomalousdiffusion equation, Appl. Math. Model., 38 (2014), pp. 3802-3821.

[15] Z. Li, Z. Liang, and Y. Yan, High-order numerical methods for solving time fractional partial differential equations, J. Sci. Comput., 71 (2017), pp. 785-803.

[16] Y. Lin and C. Xu, Finite difference/spectral approximations for the time-fractional diffusion equation, J. Comput. Phys., 225(2007), pp. 1533-1552.

[17] Ch. Lubich, I. H. Sloan, and V. Thomée, Nonsmooth data error estimates for approximations of an evolution equation with a positive-type memory term, Math. Comp., 65(1996), pp. $1-17$.

[18] W. McLean and K. Mustapha, Convergence analysis of a discontinuous Galerkin method for a fractional diffusion equation, Numer. Algorithms, 52(2009), pp. 69-88.

[19] W. McLean and K. Mustapha, Time-stepping error bounds for fractional diffusion problems with non-smooth initial data, J. Comput. Phys., 293(2015), pp. 201-217.

[20] K. Mustapha, An implicit finite difference time-stepping method for a sub-diffusion equation, with spatial discretization by finite elements, IMA J. of Numer. Anal., 31(2011), pp. 719739.

[21] K. Mustapha and W. McLean, Uniform convergence for a discontinuous Galerkin, timestepping method applied to a fractional diffusion equation, IMA J. of Numer. Anal., 32(2012), pp. 906-925.

[22] K. Mustapha and W. McLean, Superconvergence of a discontinuous Galerkin method for fractional diffusion and wave equations, SIAM J. Numer. Anal., 51(2013), pp. 491-515.

[23] K. Sakamoto and M. Yamamoto, Initial value/boundary value problems for fractional diffusionwave equations and applications to some inverse problems, J. Math. Anal. Appl., 382(2011), pp. 426-447, 2011.

[24] M. Stynes, E. O'riordan, and J. L. Gracia, Error analysis of a finite difference method on graded meshes for a time-fractional diffusion equation, Submitted for publication (2016).

[25] V. Thomée, Galerkin Finite Element Methods for Parabolic Problems, Springer - Verlag, Berlin, 2007.

[26] Y.-N. Zhang, Z.-Z. Sun and H.-L. Liao Finite difference methods for the time fractional diffusion equation on non-uniform meshes, J. Comput. Phys. 265(2014), pp. 195-210.

5. Appendix. In this Appendix, we will give the proof of Lemma 2.4. To do this, we need to introduce the polylogarithm function

$$
\operatorname{Li}_{p}(z)=\sum_{j=1}^{\infty} \frac{z^{j}}{j^{p}} .
$$

The polynomial function $\operatorname{Li}_{p}(z)$ is well defined for $|z|<1$ and $p \in \mathbb{C}$. It can be analytically continued to the split complex plane $\mathbb{C} \backslash[1,+\infty)$; see Flajolet [6]. With $z=$ 1 , it recovers the Riemann zeta function $\varsigma(p)=\mathrm{Li}_{p}(1)$. We also recall an important singular expansion of the function $\operatorname{Li}_{p}\left(e^{-z}\right)$ (Flajolet [6, Theorem 1]).

Lemma 5.1. ([10, Lemma 3.2]) For $p \neq 1,2, \ldots$, the function $L i_{p}\left(e^{-z}\right)$ satisfies the singular expansion

$$
L i_{p}\left(e^{-z}\right) \sim \Gamma(1-p) z^{p-1}+\sum_{l=0}^{\infty}(-1)^{l} \varsigma(p-l) \frac{z^{l}}{l !}, \quad \text { as } z \rightarrow 0
$$

where $\varsigma(z)$ denotes the Riemann zeta function. Then

LEMMA 5.2. ([10, Lemma 3.4]) Let $|z| \leq \frac{\pi}{\sin \theta}$ with $\theta \in\left(\frac{\pi}{2}, \frac{5 \pi}{6}\right)$ and $-1<p<0$.

$$
L i_{p}\left(e^{-z}\right)=\Gamma(1-p) z^{p-1}+\sum_{l=0}^{\infty}(-1)^{l} \varsigma(p-l) \frac{z^{l}}{l !}
$$

converges absolutely. 
Proof. [Proof of Lemma 2.4] We have, by the weights in (2.5), with $\zeta=e^{-z k}$,

$$
\begin{aligned}
\tilde{w}(z) & =\sum_{j=0}^{\infty} w_{j} \zeta^{j}=\frac{1}{\Gamma(1+\alpha)}\left(\zeta^{-1}-2+\zeta\right) \sum_{j=1}^{\infty} j^{\alpha} \zeta^{j} \\
& =\frac{1}{\Gamma(1+\alpha)}\left(\left(e^{-z k}\right)^{-1}-2+e^{-z k}\right) \operatorname{Li}_{-\alpha}(\zeta),
\end{aligned}
$$

where, by Lemma 5.2,

$$
\mathrm{Li}_{-\alpha}(\zeta)=\mathrm{Li}_{-\alpha}\left(e^{-z k}\right)=\Gamma(1+\alpha)(z k)^{-\alpha-1}+\sum_{l=0}^{\infty}(-1)^{l} \varsigma(\alpha-l) \frac{(z k)^{l}}{l !} .
$$

By (2.11), we have

$$
z_{k}^{\alpha}=\left(\frac{\delta(\zeta)}{k}\right)^{\alpha}=\frac{\tilde{w}(\zeta)^{-1}(1-\zeta)}{k^{\alpha}}=\frac{1}{k^{\alpha} \psi(z k)},
$$

where

$$
\psi(z k)=\frac{1}{\Gamma(1+\alpha)}\left(e^{z k}-1\right) \operatorname{Li}_{-\alpha}\left(e^{-z k}\right) .
$$

Using [19, Lemma 1] we may write, with $C_{\alpha}=\frac{\pi}{\sin (\pi \alpha)}$ and $z k=\rho e^{i \theta}=r+i \phi$,

$$
\begin{aligned}
\psi(z k) & =\frac{1}{\Gamma(1+\alpha)}\left(e^{z k}-1\right) \operatorname{Li}_{-\alpha}\left(e^{-z k}\right)=\frac{1}{C_{\alpha}} \int_{0}^{\infty} \frac{s^{-\alpha}}{1-e^{-z k-s}} \frac{1-e^{-s}}{s} d s \\
& =\frac{1}{C_{\alpha}} \int_{0}^{\infty} \frac{s^{-\alpha}}{1-e^{-z k-s}} \frac{1-e^{-s}}{s} d s=\frac{1}{C_{\alpha}} \int_{0}^{\infty} \frac{s^{-\alpha}}{1-e^{-r-i \phi-s}} \frac{1-e^{-s}}{s} d s \\
& =\frac{1}{C_{\alpha}} \int_{0}^{\infty} \frac{s^{-\alpha}}{1-e^{-r-s}(\cos \phi-i \sin \phi)} \frac{1-e^{-s}}{s} d s \\
& =\frac{1}{C_{\alpha}} \int_{0}^{\infty} \frac{\left(s^{-\alpha-1}\left(1-e^{-s}\right)\left(1-e^{-r-s} \cos \phi\right)\right)-\left(s^{-\alpha-1}\left(1-e^{-s}\right)\left(e^{-r-s} \sin \phi\right)\right) i}{1-2 e^{-r-s} \cos \phi+e^{-2 r-2 s}} d s
\end{aligned}
$$

which implies that

$$
z_{k}^{\alpha}=\frac{C_{\alpha}}{k^{\alpha}} \frac{1}{A-B i}=\frac{C_{\alpha}}{k^{\alpha}} \frac{A+B i}{A^{2}+B^{2}}
$$

where

$$
\begin{aligned}
& A=\int_{0}^{\infty} \frac{\left(s^{-\alpha-1}\left(1-e^{-s}\right)\left(1-e^{-r-s} \cos \phi\right)\right)}{1-2 e^{-r-s} \cos \phi+e^{-2 r-2 s}} d s \\
& B=\int_{0}^{\infty} \frac{\left(s^{-\alpha-1}\left(1-e^{-s}\right)\left(e^{-r-s} \sin \phi\right)\right)}{1-2 e^{-r-s} \cos \phi+e^{-2 r-2 s}} d s .
\end{aligned}
$$

Therefore

$$
\Re\left(z_{k}^{\alpha}\right)=\frac{C_{\alpha}}{k^{\alpha}} \frac{A}{A^{2}+B^{2}}, \quad \Im\left(z_{k}^{\alpha}\right)=\frac{C_{\alpha}}{k^{\alpha}} \frac{B}{A^{2}+B^{2}} .
$$

Let us first consider the case for $\theta=\frac{\pi}{2}$. In this case, we have, with $r=\rho \cos \theta=$ $0, \phi=\rho \sin \theta=\rho$,

$$
\Re\left(z_{k}^{\alpha}\right)=\frac{C_{\alpha}}{k^{\alpha}\left(A^{2}+B^{2}\right)} \int_{0}^{\infty} \frac{s^{-\alpha-1}\left(1-e^{-s}\right)\left(1-e^{-s} \cos \rho\right)}{1-2 e^{-s} \cos \rho+e^{-2 s}} d s .
$$


Note that

$$
1-2 e^{-s} \cos \rho+e^{-2 s}>1-2 e^{-s}+e^{-2 s}=\left(1-e^{-s}\right)^{2} \geq 0
$$

and

$$
1-e^{-s} \cos \rho>1-e^{-s}>0
$$

we get $\Re\left(z_{k}^{\alpha}\right) \geq 0$ which implies that $z_{k}^{\alpha} \in \Sigma_{\theta_{0}}$ for any $\theta_{0} \in\left(\frac{\pi}{2}, \pi\right)$. Now let us choose $\theta$ close to $\frac{\pi}{2}, \theta>\frac{\pi}{2}$. By the continuity of $z_{k}^{\alpha}$ with respect to $\theta$, [10, Proof of Lemma 3.6], there exists $\theta_{0} \in\left(\frac{\pi}{2}, \pi\right)$ such that

$$
z_{k}^{\alpha} \in \Sigma_{\theta_{0}} \text { for all } z \in \Gamma_{\theta}
$$

Together these estimates complete the proof of Lemma 2.4.

( 